\title{
AReViRoad: a virtual reality tool for traffic simulation
}

\author{
D. Herviou \& E. Maisel \\ European Center of Virtual Reality, Brest, France
}

\begin{abstract}
In this article we introduce AReViRoad, a virtual reality tool: a traffic road simulator that enables one to determine the impact of the road network on traffic fluidity. The traffic generation is based on a multi-agent system in which each vehicle (an entity) is a software agent with its own behavior (autonomous). This solution offers a credible traffic road that will be used for testing a road network. With a set of given tools one can create one's own road network. Thereafter, one can configure one's own simulation (number of vehicles, tested road network, flow of car generators) and then launch, observe and analyse it with dedicated tools, such as a $3 \mathrm{D}$ view, flow meter and timer.

Keywords: traffic road simulation, virtual reality, virtual actors, conception and analysis of urban road networks.
\end{abstract}

\section{Introduction}

The increase of vehicles in urban areas implies traffic congestion. To reduce traffic jams, several solutions exist; some are not very expensive i.e. smart traffic lights, but others are i.e. road network modification. This last solution needs to be tested and validated prior to its application. Tools that help to conceive it rely notably on traffic road generation and software for creating the road network.

In this article, we introduce the AReViRoad- a traffic road simulator based on a microscopic simulation. We will first explain the existing solutions for generating a traffic road and then detail the one we have chosen by exploring the driver representation inside AReViRoad. Next we will see the simulation environment and the dedicated tools used to build them. Finally we will show two examples of the usage for AReViRoad: the test of two different configurations of road networks and their impacts on global traffic fluidity and a smart traffic light system used to regulate traffic flow. 
We will conclude this article by mentioning the current and future developments and the other opportunities this work offers in several domains.

\section{Previous works}

The need for a traffic road to test the impact of a road network topology is obvious, which is why in this section we explain the solutions used to generate or simulate the traffic of vehicles. The common goal of these solutions is to simulate more or less precisely the temporal evolution of the traffic road: which is why criteria exist to classify them.

\subsection{Generation of traffic flow}

If we look at the level of detail inside the simulation, we find three families: the macroscopic, the mesoscopic and the microscopic simulation.

The macroscopic simulations consider the vehicles as an indivisible whole. The principle is inspired by hydrofluid dynamics and has been retranscribed by analogy to a flow of vehicles and a road network [1]. The simulation of traffic evolution consists of the evaluation of these equations. The time of computation for a state of the system is independent of the number of simulated vehicles. Nevertheless, it is very difficult, even impossible, to modify parameters during the simulation without having to launch it again.

The mesoscopic simulations consider each vehicle as an independent element of the simulation. Each of them has its own intrinsic properties. However, in these simulations the actions carried out by vehicles are instantaneous. This is notably due to the fact that space is often sliced into cells: the action of changing lane on the road can only be achieved by a cell change. This kind of simulation can be obtained, for example, with models based on cellular automata [2] and is mainly used for the prediction of traffic jams on highways.

The microscopic simulation also considers each vehicle independently. The possible actions are generally refined. Here, changing from one lane to another is a continuous control process on the vehicle that can take into consideration security rules. The traffic obtained with this kind of simulation is relatively credible from the observation point of view. Moreover, this approach offers good flexibility and it is often possible to insert new constraints during online simulation and to interact with the traffic flow. For these reasons the solution is not exact [3], notably in domains that require precision at the microscopic scale as driving simulations.

Other criteria can be used to classify the different approaches as the temporal model used (continuous time or discrete event) or the prediction model used (determinist or stochastic).

We have chosen the microscopic approach because we have considered it as the most adapted for testing a road network of the size of several blocks. Indeed, this case requires precision for the generated traffic flow as, for example, being able to simulate consequences of a one lane roundabout or a two lane roundabout on global fluidity. This implies the use of traffic flow generation that relies on 
virtual drivers that are representatives of society. In computer science, different solutions have been proposed to accomplish this difficult task. Among them, there are cognitivist approaches [4], computational and reactive approaches [5] or emotional-based approaches [6].

\section{AReViRoad: traffic road simulation}

\subsection{Simulation environment}

Our simulator is made of a road network, a scheduler and a $3 \mathrm{D}$ engine. The road network consists of a matrix of sections. Among them we could find a multi-lane straight line, multi-lane roundabout or a crossroad. This network also has urban furniture: buildings, bus station, parking. Finally, the traffic signs complete the road network environment. The virtual drivers and pedestrians use it as their environment of evolution. The activities inherent to this evolution are offered by the real time scheduler that governs the parallel execution of each autonomous actor: they can perceive, decide and act with their own behavior. The graphic engine computes the 3D visualization of the virtual world and enables the observation of traffic road evolution in real time from different points of view.

\subsection{Traffic flow generation}

Next we will explain the key-point of the traffic road generation. As written above, the traffic generation lies on the autonomous evolution of virtual drivers, consequently we will detail how they are designed and what they do during their autonomous evolution.

\subsubsection{The driving tasks}

In order to model a driver, we have adopted the three parts model of Michon [7]. This model determines the drivers' tasks as follows: a strategical part (road plan), a tactical part (lane choice) and an operational part (steering angle).

The tactical and operational parts of the model are described using degraded driving tasks. A driving task is an action plan that describes what to do (visuals information acquisition, actions on vehicle) in a typical given situation with the respect of spatio-temporal constraints. For example, crossing a crossroads, going outside a parking place are driving tasks. This solution is well suited to conceive reactive virtual drivers, because it expresses the data in a manner close to the one used to program in computer science (events, procedures,...). Driving tasks are implemented using automata.

\subsubsection{Perception}

To make decisions at the tactical and strategical level, virtual drivers need information about their environment. They have to perceive the roadsigns, the other drivers, the bikes, the pedestrians... but in a realistic way. Actually, the virtual drivers do not have to see everything because real drivers do not [8]. 
We have assumed that a lot of mistakes made during driving came from bad visual information acquisition. Consequently, we focus part of our work on the conception of a perception model based on visual routines. A visual routine is an automated time constrained movement of the eyes done to acquire information of the environment [9], for example: when crossing a crossroad straight: look left, right, left and ahead. The visual information perceived is stored in a visual memory that will be used for decisions.

\subsubsection{Decisions}

The decision of a virtual driver relies on two parts: the driving task and the three parts determined above. At the strategical level, the driver determines its global navigation, this gives input information for the tactical level that determines which direction to take at an intersection and computes the trajectory to follow. Now that the global trajectory is known, the virtual driver has to adapt their speed on this track according to what they have perceived of their environment and a simplified set of highway rules. Once a decision is taken, the according "high level" action has to be implement: reach a speed, stop at given point, keep speed...

\subsubsection{Actions}

The action part has to implement the action chosen at the decision level: this is the operational part work. This one receives as input a "high level" action to do: follow trajectory, keep speed, follow vehicle, stop at a point... and gives has output comprehensive signals for the car: braking force, accelerating force, steering angle...

\section{AReViRoad: tools for creation and evaluation}

\subsection{Design its own environment}

AReViRoad offers several tools to conceive the environment of simulation. The first one (see left on figure 1) is the road network editor. For simplicity in the road network conception we have at the moment considered it as a matrix of customizable sections. A library of predefined sections is available: one or two lanes straight, curved, crossroad or roundabout sections. Even a person unfamiliar with the software can easily build his road network.

The second one is the customizing section: adding urban furniture, buildings, park cars on sections (see center on figure 1). It is important to consider this phase in the conception of the road network, because virtual drivers will not be able to see through obstacles and will consequently adapt their behavior to the situation (for example: more time will be needed to check for vehicles presence). Moreover, if modifying the road network implies for example to remove or add a building the designer can see directly the consequences in terms of visibility .

The third one is for putting the customized sections on the road network created with the first tool in order to obtain a final road network as the one visible on figure 1 right. This one also offers the possibility of adding traffic signs as stop 
or traffic lights. In order for the tester to look at strategical locations on the road network during simulations it is possible to register predefined positions of virtual cameras.

Finally, an xml file is used to configure a simulation. We can notably define the number of vehicles and the road network used.
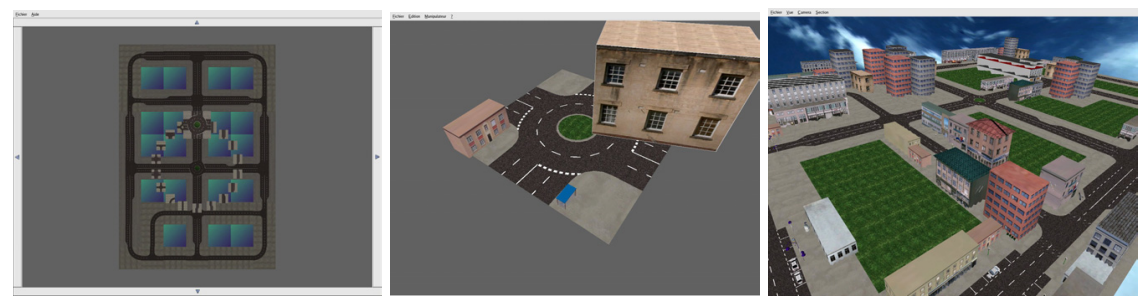

Figure 1: A set of tools are available to create the road network topology, the section look and the final usable road network.

\subsection{Evaluation of road network}

During the simulation we offer several tools: a car generator that can be used to input vehicles on the road network at a variable flow rate, a flow meter to determine the number of vehicles per hour at a location, and if the road network is opened a counter gives the virtual driver life duration which computes from its insertion on the road network until it goes outside.

\section{Experiments and results}

\subsection{Time to pass through city}

The first example is a very simple test done on two different road network configurations: the objective is to determine the consequences of changing the first road network into the second one (see figure 2) in term of global traffic flow (time spent on the road network) and the limit of each one (when congestion appears). This study case can be correlated to the one that tries to determine if changing a two lanes boulevard into one lane for vehicles and one lane dedicated for buses will impact on traffic fluidity and in which proportion.

The two roads networks are opened. This means that not all roads are linked, which is the same as the part of a city we have focused on. The road networks are composed of straight sections and two right priority crossroads that give about one kilometer of road. In the first one the straight roads are one lane, in the second one a boulevard has been created with two lanes of straight roads: this axis is considered the main axis on the two road networks. As the road networks are opened we need automatic car generation to build a traffic flow. Thus we have car generators placed 
at each road network input (six on the tested ones). Each one can be parameterized to generate a given number of vehicles per hour.

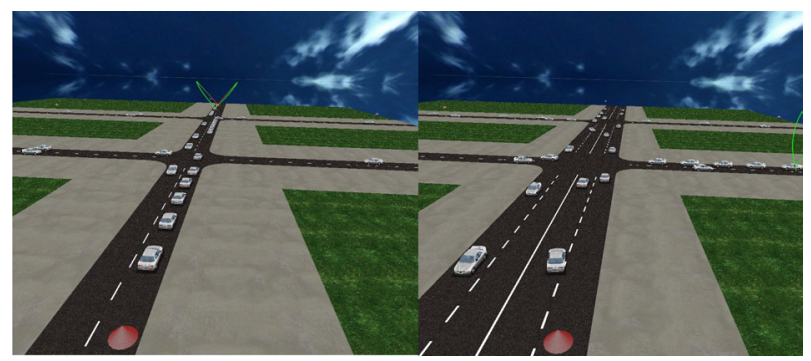

Figure 2: Left road network is the first one to be tested: a one lane straight road as main axis. Right road network has a two lanes straight road as main axis. Red spot at bottom left on each picture is one of the six car generators.

\subsection{Experimentations}

The tests are done as follows: each road network is tested with a different flow rate for each car generator: 100 to 600 vehicles/hour (v/h), and these flow rates are changed during online simulation. In our simulation we have also fixed all car generators at the same flow rate and excluded the ones placed on the main axis that have a speed up factor of one and a half (150 to $900 \mathrm{v} / \mathrm{h}$ ). We do not consider the travel of each vehicle but only its time spent on the road network: to measure the global time a car spend on the road network, we use their life time. The results of these two simulations are visible in figure 3.

\subsection{Results}

The global average time is constant and almost the same for both road networks until the density value of 35 vehicles per kilometer $(\mathrm{v} / \mathrm{k})$. After this limit the first road network seems to be more sensitive to an increase in the vehicle density. This observation can be correlated with the standard deviation visible on figure 3 bottom right. At this same value, the time to pass through the road network offers an interval [minimum,average+sigma] that increases rapidly: the road network capacity of absorbtion has almost been reached. The same phenomenon is observed later on the second road network for a density value of $95 \mathrm{v} / \mathrm{h}$ and is confirmed by both average time and interval time increasing.

We can conclude that if the second road network is changed to become the first one, then to prevent congestion, the flow of cars that pass through it in reality have to be less than its limit of congestion which seems to be about three time less.

We could have obtained more reliable results by using real input data for car generation and also the itinerary of each vehicle on the road network. 

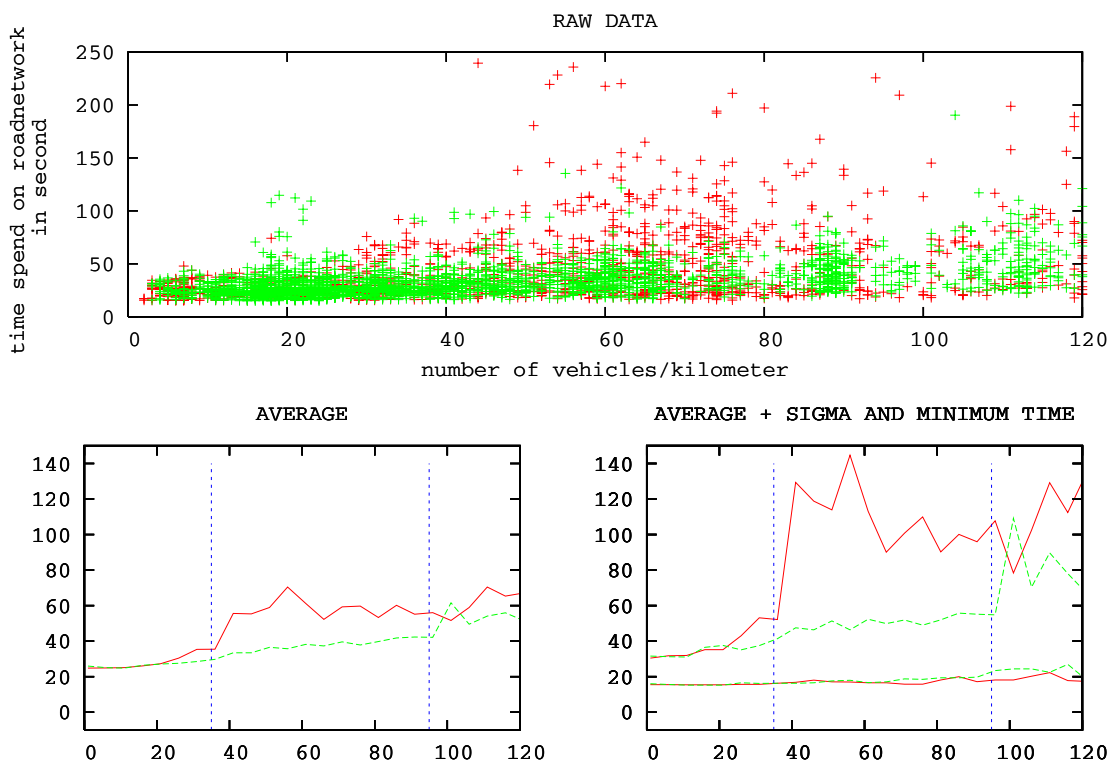

AVERAGE + SIGMA AND MINIMUM TIME

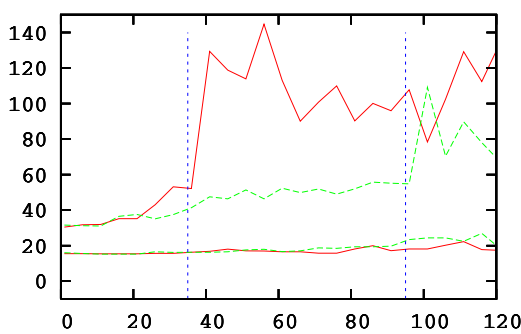

Figure 3: In red results obtain on the first road network, in green results on the second one. Top diagram show raw data: each point gives the life time of a car function of the density of vehicle on the road network when it leaves it. Bottom left diagram: average time spend on the road network. Bottom Right diagram: minimum time and average time plus sigma (standard deviation).

Moreover, the study considers that road network outputs are never congested and consequently do not influence the creation of traffic jam on the road network.

\subsection{The smart traffic light}

The second test is described briefly here and more explanations can be found in [10].

We have tested the impact of a smart traffic lights on average waiting time (awt) at a crossroad. A smart traffic lights detects the presence of vehicles on a lane and if the orthogonal way is free of vehicles it switches the corresponding light to green. The detection of vehicles is done with virtual sensors that simulate a camcorder or telemeter. The test was done on a simple close road network with twelves crossroads on a surface of 300 meters by 300 meters. Results show that 
the awt with a managed switching light is far less than the awt without, but with an increase in density awt with smart traffic lights tends to be similar to awt without: this confirms the first intuition.

This example is just to show that it is also possible to test an intelligent transport system and to view the impacts on road network fluidity. It becomes possible to test an algorithm that take into account communication between traffic lights and vehicles or that considers the density of vehicles or that detects emergency vehicles (fire fighters, ambulance).

\section{Conclusion and perspectives}

We have introduced AReViRoad, a traffic road simulator and a set of tools dedicated to conceive its own simulations: road networks creation, configuration and test. Based on a multi-agents system in which each virtual driver perceives, decides and acts on its vehicle, it enables credible traffic road generation.

It becomes possible to visualize, analyse and modify simulations easily, to conceive the best road network in terms of traffic road fluidity. AReViRoadseems to be an adapted tool to test road network at a center city scale due to the microscopic simulation on which it relies: it is possible to determine at a global scale the impact of a roundabout instead of a traffic light at a crossroads.

Today's developments concern the diversification of driver behaviors to increase credibility of generated traffic road. Thereafter, we will investigate the validation of virtual drivers behavior to increase reliability of results obtain in simulation and the development of a tool dedicated to the creation of more complex virtual road networks to fit with real ones.

\section{References}

[1] I. Bonzani. Hydrodynamic models of traffic flow: Drivers' behaviour and nonlinear diffusion. Mathematical and Computer Modelling, 31(6-7):1-8, March-April 2000.

[2] Sven Maerivoet and Bart De Moor. Cellular automata models of road traffic. Physics Reports, 419(1):1-64, November 2005.

[3] Alexis Champion, René Mandiau, Christophe Kolski, Alexandre Heidet, and Andras Kemeny. Traffic generation with SCaNeR(c) II simulator : Towards a multi-agent architecture. In Proceedings of DSC'99, 1999.

[4] D. Krajzewicz and P. Wagner. ACME (A Common Mental Environment)driver. a cognitive car driver model. In Meuth Amborski, Krzysztof and Hermann, editors, proceedings of the 16th Simulation Multiconference ”Modelling and Simulation 2002", pages 689-693, june 2002.

[5] Douglas Reece and Steven Shafer. A computational model of driving for autonomous vehicles. Technical Report CMU-CS-91-122, Computer Science Department, Carnegie Mellon University, Pittsburgh, PA, April 1991. 
[6] T. Al-Shihabi and R. R. Mourant. A framework for modeling humanlike driving behaviors for autonomous vehicles in driving simulators. In Proceedings of the fifth international conference on Autonomous agents, may 2001.

[7] J. Michon. A critical view of driver behavior models : what do we know, what should we do ? In Human behavior and traffic safety. L. Evans, R. Schwing, 1985.

[8] R. Van Rullen and C. Koch. Competition and selection during visual processing of natural scenes and objects. Journal of Vision, (3):75-85, 2003.

[9] Mary M. Hayhoe, Dana H. Ballard, Hiroyuki Shinoda Jochen J. Triesch, Pilar Aivar, and Brain T. Sullivan. Vision in natural and virtual environments. Eye Tracking Reasearch and Applications Symposium, pages 7-13, 2002.

[10] Herviou David and Maisel Eric. AReViRoad : a traffic simulator to test intelligent transportation systems. In Proceedings of the $5^{\text {th }}$ International Conference on Intelligent Transportation Systems and Telecommunications, pages 193-196, June 2005. 